\title{
Chromosome aneuploidy analysis in embryos derived from in vivo and in vitro matured human oocytes
}

Jianhua $\mathrm{Li}^{1 \dagger}$, Jing Chen ${ }^{2,3+}$, Tiecheng Sun ${ }^{4}$, Shuiwen Zhang ${ }^{1}$, Tingting Jiao ${ }^{1}$, Ri-Cheng Chian ${ }^{*}$, Youzhu Li ${ }^{2^{*}+}$ and YeXu⿰氵 ${ }^{1 *+}$

\begin{abstract}
Background: In vitro oocyte maturation (IVM) is being increasingly approached in assisted reproductive technology (ART). This study aimed to evaluate the quality of embryos generated by in-vitro matured immature follicles, as a guideline for further clinical decision-making.

Methods: A total of 52 couples with normal karyotypes underwent in vitro fertilization, and 162 embryos were donated for genetic screening. Embryos in IVF group were generated by mature follicles retrieved during gonadotrophin-stimulated in vitro fertilization (IVF) cycles. And embryos in IVM group were fertilized from IVM immature oocytes.
\end{abstract}

Results: The average age of the women was $30.50 \pm 4.55$ years (range 21-42 years) with 87 embryos from IVF group and 75 embryos from IVM group. The rate of aneuploid with 28 of the 87 (32.2\%) embryos from IVF group and 21 of the 75 (28\%) embryos from IVM group, with no significant difference. The frequency of aneuploid embryos was lowest in the youngest age and increased gradually with women's age, whether in IVF group or IVM group and risen significantly over 35 years old. The embryos with morphological grade 1 have the lowest aneuploidy frequency (16.6\%), and increase by the grade, especially in IVF group. In grade 3, embryos in IVM group were more likely to be euploid than IVF group (60\% vs 40\%, respectively).

Conclusions: IVM does not affect the quality of embryos and does not increase the aneuploidy rate of embryos. It is clinically recommended that women more than 35 years have a high aneuploidy rate and recommended to test by PGS (strongly recommended to screened by PGS for women more than 40 years). Women aged less than 35 years old for PGS according to their physical and economic conditions. Embryo with poor quality is also recommended to test by PGS, especially for grade III embryos.

*Correspondence: rchian@126.com; liyouzhu2006@sina.com; |1421276844@163.com

${ }^{\dagger}$ Jianhua Li and Jing Chen contributed equally to this work

${ }^{1}$ Reproductive Medical Center, Senior Department of Obstetrics \& Gynecology, The Seventh Medical Center of PLA General Hospital, Beijing 100700, China

${ }^{2}$ Reproductive Medicine Center, The First Affiliated Hospital of Xiamen University, No. 6 Guchengxi Road, Si Ming, Xiamen 361003, China

${ }^{5}$ Center for Reproductive Medicine, Shanghai Tenth People's Hospital of Tongji University, Shanghai 200072, China

Full list of author information is available at the end of the article

\section{Background}

A growing number of couples adopt assisted reproductive technologies (ART) in infertility clinics to conceive a child. Due to the dominant follicles in follicular development may suppress the small follicles' growth, nearly $15 \%$ of oocytes retrieved by gonadotrophin-stimulated original author(s) and the source, provide a link to the Creative Commons licence, and indicate if changes were made. The images or other third party material in this article are included in the article's Creative Commons licence, unless indicated otherwise in a credit line to the material. If material is not included in the article's Creative Commons licence and your intended use is not permitted by statutory regulation or exceeds the permitted use, you will need to obtain permission directly from the copyright holder. To view a copy of this licence, visit http://creativecommons.org/licenses/by/4.0/. The Creative Commons Public Domain Dedication waiver (http://creativeco mmons.org/publicdomain/zero/1.0/) applies to the data made available in this article, unless otherwise stated in a credit line to the data. 
Keywords: In vitro oocyte maturation (IVM), In vitro fertilization (IVF), Embryo, Aneuploidy, Preimplantation genetic screening (PGS)

in vitro fertilization (IVF) cycles are immature [1], and these immature oocytes are usually discarded. However, in controlled ovarian hyperstimulation $(\mathrm{COH})$ cycle high yield of follicle may increase the risk of complications, such as: ovarian hyperstimulation syndrome (OHSS), torsion of ovarian pedicle, and intra-abdominal bleeding [2]. Furthermore, Baart et al. suggested that high-dose of gonadotrophin-stimulated in $\mathrm{COH}$ cycle may lead to high frequency of aneuploidy [3]. In vitro maturation (IVM) of immature follicles as an alternative method which has become a promising strategy to maximize the utilization of follicles and maximum cost savings, it could be beneficial for women with polycystic ovaries, poor ovarian response to gonadotropin treatment, premature ovarian failure, and cryopreserve their oocytes before monotherapy to preserve their fertility [4].

The procedure and the techniques used for IVM is no generally accepted and substantially different across infertility clinics. The quality of mature oocyte is the key to embryo quality and developmental potential leading to chromosomal anomalies or embryo loss. Therefore, ensuring couples conceive in decrease time with healthy embryo is important through genetic analysis of embryo for reproductive medicine. For human embryos, aneuploidy is of great relevance to embryo selection. The most common type of genetic abnormality and the leading cause of miscarriage, implantation failure is aneuploidy [5]. More than half of embryos produced by IVF cycles are aneuploid [6].

IVM of immature oocytes, recovered from natural cycle, is a useful cost-effective treatment for infertility in women. Whereas, there was no detectable comprehensive evidence on the frequency of aneuploidy on these embryos based on IVM of immature oocytes retrieval. As well, the relationship between genetic status and embryo morphology remains unclear. Preimplantation genetic screening (PGS) for embryos has gradually become a gold criterion in infertility treatment, offer a more accurate assessment of chromosome status, not only to enhance the implantation and pregnancy rates, but also to improve the healthy offspring, such as: reduce miscarriages, risk of aneuploid offspring, and time to conceive. We aimed to compare the quality of embryos between IVM and IVF groups through genetic screening, for further clinical decision-making.

\section{Materials and methods Study design and patients}

This study was reviewed and approved by the Ethics Committee at The Seventh Medical Center of PLA General Hospital (Research License 2021-31). All embryos were obtained from donors at the Center of Reproductive Medicine in The Seventh Medical Center of PLA General Hospital. Recruitment took place from April 2005 to February 2015.

Written informed consent was provided before donating their surplus frozen embryos for research. The donors were financially compensated for the effort, time and inconvenience related to the donation process. A total of 162 embryos donated from 52 couples with normal karyotypes were used in this study. All of the women with normal ovaries, uterus, and regular menstrual cycles had normal basal FSH levels $(<10 \mathrm{mIU} / \mathrm{mL}$ on day 3 of the menstrual cycle). We performed preimplantation genetic screening (PGS) for chromosome aneuploidy analysis in embryos derived from in vivo (IVF group) and in vitro matured human (IVM group) oocytes.

\section{Controlled ovarian hyperstimulation (COH) in IVF group}

For patients with $\mathrm{COH}$ cycle, the treatment was initiated from a baseline transvaginal ultrasound scan on day 3 of the menstrual cycle to ensure that there were more than seven small antral follicles in both ovaries. Ovary stimulation was carried out with exogenous gonadotropins after a desensitization protocol with GnRH analogues according to a long or a short protocol, depending on the patient's previous history of gonadotropin response and other factors. An ultrasound scan was performed on day 7-9 and subsequently until one follicle reached $18 \mathrm{~mm}$ and two reached $16 \mathrm{~mm}$ in diameter, and then 10,000 IU human chorionic gonadotropin (hCG) was administered. Oocyte retrieval was performed approximately $36 \mathrm{~h}$ after hCG administration.

\section{Follicle retrieval and in-vitro maturation (IVM)}

IVM is routinely performed in our center for patients who had normal ovaries with $>7$ antral follicles in both ovaries. Briefly, the treatment was initiated from a baseline transvaginal ultrasound scan on day 3 of the menstrual cycle to ensure that there were more than seven small antral follicles in both ovaries. On day 7-9 ultrasound scans were repeated. When the dominant follicle reached $12-14 \mathrm{~mm}$ in diameter and/or the endometrial 
thickness was $\geq 6 \mathrm{~mm}$, hCG $(10,000 \mathrm{IU})$ was administered and oocyte retrieval was performed $36 \mathrm{~h}$ later.

The oocyte retrieval was performed using transvaginal ultrasound-guided aspiration was performed with a 17-gauge double-lumen needle (Cook, Eight Mile Plains, Queensland, Australia) for aspiration of the leading follicles. A 19-gauge single-lumen needle (Cook) for the small follicles. For aspiration, a portable pump was connected to the needle with a pressure of $<100 \mathrm{~mm} \mathrm{Hg}$ for leading follicles and $<40 \mathrm{~mm} \mathrm{Hg}$ for small follicles. After assessing the nuclear maturity of the retrieved oocytes under the dissecting microscope as the commonly used method [7]. The collected matured oocytes were inseminated 2 or $3 \mathrm{~h}$ later by intracytoplasmic sperm injection (ICSI), while the collected immature oocytes were cultured in IVM medium.

The immature oocytes at Metaphase-I (MI) and GV stage were cultured in a $1 \mathrm{~mL}$ maturation medium containing $30 \%$ serum of the patient's own (inactivated at $56^{\circ}$ for $30 \mathrm{~min}$ ) with $75 \mathrm{mIU} / \mathrm{mL}$ human FSH ((Gonal-F; Merck Serono, Switzerland), $75 \mathrm{mIU} / \mathrm{ml}$ human menopausal gonadotrophin (hMG, Lizhu Pharmacy) and $10 \mathrm{ng} / \mathrm{ml}$ recombinant human epidermal growth factor (Invitrogen, Carlsbad, CA, USA) to induce final oocyte maturation at $37{ }^{\circ} \mathrm{C}$ in $5 \% \mathrm{CO}_{2}, 5 \% \mathrm{O}_{2}$ and $90 \% \mathrm{~N}_{2}$ with high humidity for 24 or $48 \mathrm{~h}$. Then the oocytes matured in vitro were also inseminated by ICSI [8].

\section{Embryo culture}

All embryos were fertilized using ICSI. The zygotes were cultured in individual $20-\mu \mathrm{L}$ droplets of G1-PLUS medium (Vitrolife, Gothenburg, Sweden) overlaid with 2.5-mL mineral oil (Vitrolife, Gothenburg, Sweden) in a 30-mm Falcon culture dish and incubated at $37^{\circ} \mathrm{C}$ in an atmosphere containing $5 \% \mathrm{O}_{2}$ and $6.0 \% \mathrm{CO}_{2}$. On day 3 or day 4 oocyte retrieval, the embryos were vitrification for the further genetic screen.

\section{Whole-genome amplification and DNA sequencing}

After thawing, whole embryo (WE) samples were transferred into RNase- and DNase-free PCR tubes containing $5 \mu \mathrm{L}$ cell lysis buffer (Yikon Genomics, China), and frozen immediately in liquid nitrogen stored at $-80^{\circ} \mathrm{C}$ until further processing.

DNA for whole-genome amplification was amplified with the multiple annealing and looping based amplification cycles (MALBAC) technique and library generation (Cat No. YK001B, Yikon Genomics) as previously described $[9,10]$. Amplification products were sequenced on an Illumina HiSeq 2500 platform (Illumina, San Diego, CA, USA) with approximately two million sequencing reads per sample. The read numbers were counted along the whole genome with a bin size of
$1 \mathrm{Mb}$ and normalized based on GC content and a reference dataset. The number of reading counts served as the index of ploidy: a 50\% increase indicates an increase in the number of chromosomes from 2 to 3, whereas a $50 \%$ decrease indicates a reduction in the number of chromosomes from 2 to $1[11,12]$.

\section{Statistical analysis}

Continuous data were reported based on mean \pm standard deviations. Data analysis was performed using SPSS (version 20). The frequency between the groups were assessed using Fisher's exact test. Differences were considered significant at $\mathrm{P}<0.05$.

\section{Results}

A total of 162 embryos were recovered and analyzed for PGS from 53 couples. The average age of the women was $30.50 \pm 4.55$ years (range $21-42$ years) with 87 embryos from IVF group and 75 embryos from IVM group. There was no significant difference $(\mathrm{P}>0.05)$ between the two groups in euploid or aneuploid. The rate of aneuploid with 28 of the 87 (32.2\%) embryos from IVF group and 21 of the $75(28 \%)$ embryos from IVM group (Table 1; Fig. 1). Only 5.7\% triploid, $6.9 \%$ haploid, $8.0 \%$ mosaic, $4.6 \%$ fragment abnormality, and $10.3 \%$ chaotic in 87 embryos samples of IVF group vs. only $5.5 \%$ triploid, $8.0 \%$ haploid, $4.0 \%$ mosaic, $4.0 \%$ fragment abnormality, and $13.3 \%$ chaotic were also detected in 75 embryos samples of IVM group (Table 1; Fig. 1).

\section{Correlation of age with euploidy}

The rate of aneuploid embryos was lowest in the youngest age and increased gradually with women's age (Fig. 2a) Whether in IVF group or IVM group and risen significantly over 35 years old (Fig. 2b). Women below the age

Table 1 Clinical characteristics of patients for whole embryos PGS between IVF group and IVM groups

\begin{tabular}{llll}
\hline & IVF & IVM & Total \\
\hline Number of patients & 26 & 26 & 52 \\
Age (Year) & $30.96 \pm 5.74$ & $30.07 \pm 3.13$ & $30.50 \pm 4.553$ \\
Number of embryo sample & 87 & 75 & 162 \\
Euploid (\%) & $59(67.8)$ & $53(70.7)$ & $112(69.1)$ \\
Aneuploid (\%) & $28(32.2)$ & $21(28.0)$ & $59(36.4)$ \\
Triploid (\%) & $5(5.7)$ & $4(5.3)$ & $9(5.6)$ \\
Haploid (\%) & $6(6.9)$ & $6(8.0)$ & $12(7.4)$ \\
Mosaic (\%) & $7(8.0)$ & $3(4.0)$ & $10(6.2)$ \\
Fragment (\%) & $4(4.6)$ & $3(4.0)$ & $7(4.3)$ \\
Abnormality (\%) & & & \\
Chaotic (\%) & $9(10.3)$ & $10(13.3)$ & $19(11.7)$
\end{tabular}

Values shown are $\mathrm{n}, \mathrm{n}(\%)$ or mean $\pm \mathrm{SD}$, unless otherwise noted 


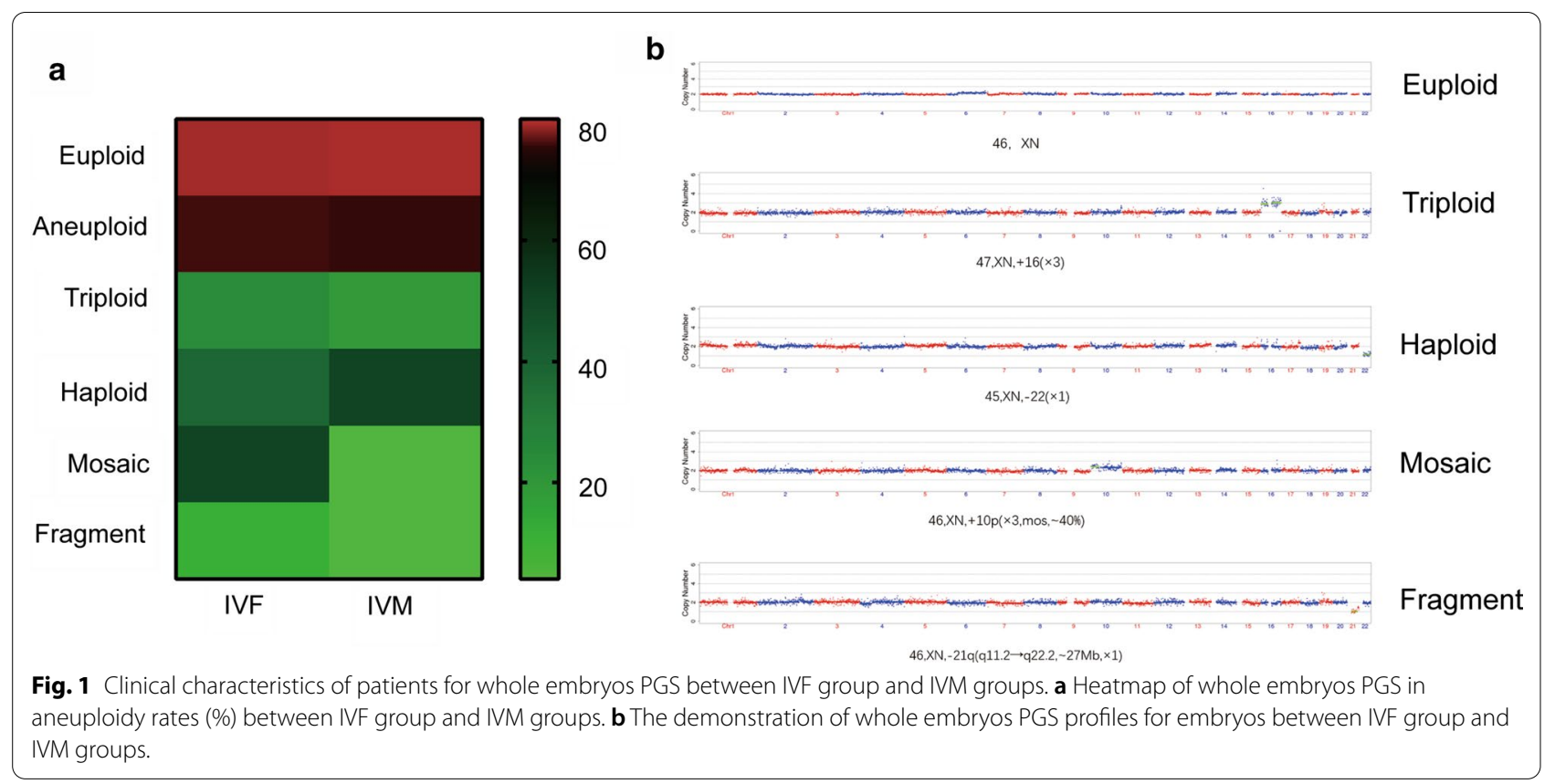

a

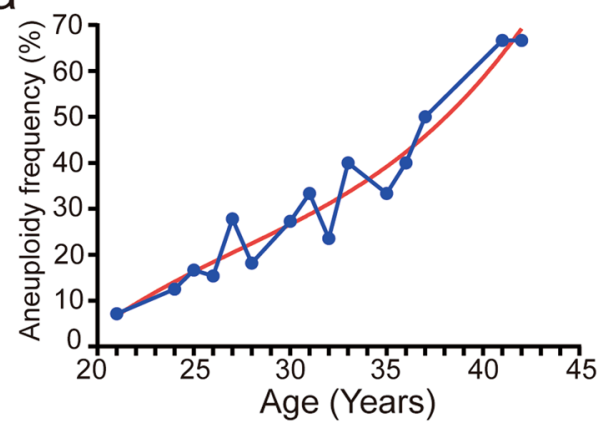

C

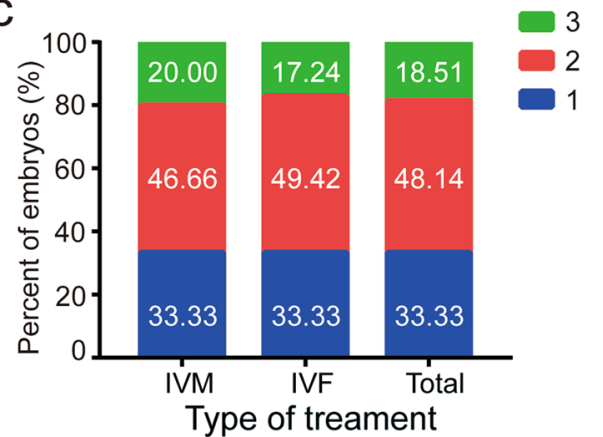

b

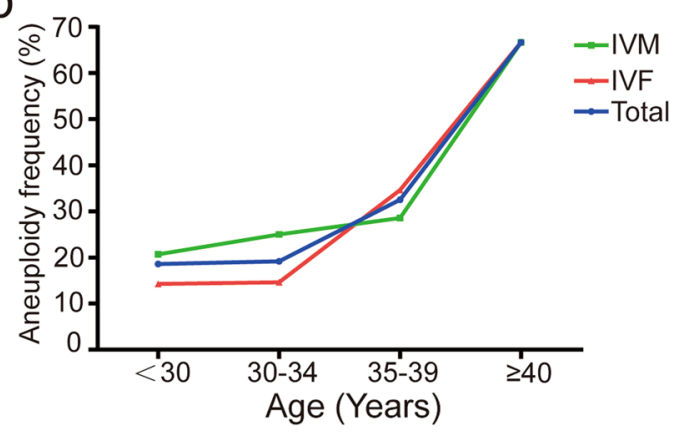

d

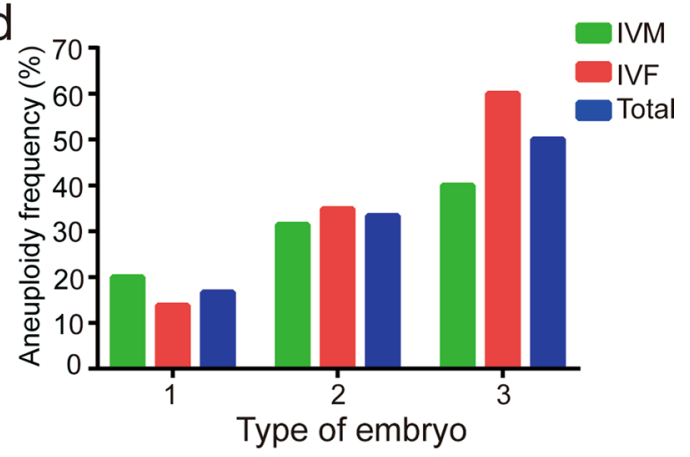

Fig. 2 Embryo aneuploidy rate according to the woman's age and morphology grade of the embryo. a The association between women's age and aneuploidy rates. Aneuploidy rates are lowest in young women and start to gradually increase with age. $\mathbf{b}$ Embryo aneuploidy rates in different age stages of women between IVF group and IVM group. No significant differences in the aneuploid rate detected between IVF group or IVM group. c The percentage in each grade of the embryo on morphology between the two groups was no significant difference. $\mathbf{d}$ The worse the embryo grade, the higher the aneuploidy rate, especially in IVF group 


\begin{tabular}{|c|c|c|}
\hline & IVF & IVM \\
\hline \multicolumn{3}{|l|}{ A } \\
\hline No. of embryos & 29 & 25 \\
\hline Euploid (\%) & $25(86.2)$ & $20(80.0)$ \\
\hline Aneuploid (\%) & 4(13.79) & $5(20)$ \\
\hline Triploid (\%) & 0 & 0 \\
\hline Haploid (\%) & $3(10.3)$ & 0 \\
\hline Mosaic (\%) & $1(3.4)$ & $1(4.0)$ \\
\hline Fragment abnormality (\%) & $1(3.4)$ & $1(4.0)$ \\
\hline Chaotic (\%) & 0 & $3(12.0)$ \\
\hline \multicolumn{3}{|l|}{ B } \\
\hline No. of embryos & 43 & 35 \\
\hline Euploid (\%) & $28(65.1)$ & $24(68.6)$ \\
\hline Aneuploid (\%) & $15(34.88)$ & $11(31.42)$ \\
\hline Triploid (\%) & $5(11.6)$ & $4(11.4)$ \\
\hline Haploid (\%) & $1(2.3)$ & $5(14.3)$ \\
\hline Mosaic (\%) & $1(2.3)$ & $2(5.7)$ \\
\hline Fragment abnormality (\%) & $3(7.0)$ & $2(5.7)$ \\
\hline Chaotic (\%) & $6(14.0)$ & $3(8.6)$ \\
\hline \multicolumn{3}{|l|}{$C$} \\
\hline No. of embryos & 15 & 15 \\
\hline Euploid (\%) & $6(40.0)$ & $9(60.0)$ \\
\hline Aneuploid (\%) & $9(60)$ & $6(40)$ \\
\hline Triploid (\%) & 0 & 0 \\
\hline Haploid (\%) & $2(13.3)$ & $1(6.7)$ \\
\hline Mosaic (\%) & $2(13.3)$ & 0 \\
\hline Fragment abnormality (\%) & $3(20.0)$ & 0 \\
\hline Chaotic (\%) & $3(20.0)$ & $4(26.7)$ \\
\hline
\end{tabular}

A, embryos of Grade 1 evaluated by morphology; B, embryos of Grade 2 evaluated by morphology; C, embryos of Grade 3 evaluated by morphology

of 35 years are nearly $20 \%$, increasing to $32.5 \%$ in 39 years old, and $66.66 \%$ for women more than 40 years. Notably, no significant differences in the aneuploid rate detected between IVF group or IVM group $(\mathrm{P}>0.05)$.

\section{Correlation of embryo morphology with euploidy}

The percentage in each grade of the embryo between the two groups was no significant difference $(P>0.05)$ (Fig. 2c). But the worse the embryo grade, the higher the aneuploidy rate, especially in IVF group (Fig. 2d). Embryos with grade 1 and 2 have a similar rate of aneuploidy both in IVM group and IVF group, but in grade III, embryos in IVM group were more likely to be euploid than IVF group ( $60 \%$ vs $40 \%$, respectively). Of the 29 embryos in IVF group assigned morphology grade 1, $13.79 \%$ were aneuploid while in IVM group ( 25 embryos) $20 \%$ were aneuploid; of the 43 embryos with morphology grade $2,34.88 \%$ were aneuploid in IVF group while $31.42 \%$ aneuploid of the 35 embryos in IVM group; and
$60 \%$ aneuploid of the 15 embryos with grade 3 in IVF group while $40 \%$ aneuploid of the 15 embryos in IVM group (Table 2).

\section{Discussion}

Recently considerable changes are afoot regarding "routine" IVF. As an increasingly interesting treatment, IVM becomes attracting attention. Primarily because the mature follicles can be directly ICSI for fresh embryo transfer; particularly the immature follicles can be cultured in vitro to increase the utilization rate of follicles; finally, the occurrence of ovarian hyperstimulation syndrome can be reduced, so that patients can improve the yield rate of follicles and also reduces the side effect of repeated ovarian stimulation with $\mathrm{GnRH}$ and gonadotropin treatment.

In the natural ovulatory cycle, small follicles are destined to be atretic with only a dominant follicle achieve maturity and ovulation. In the conventional IVF cycle, the daily use of gonadotrophins can induce many follicular developments, but dominant follicles in ovarian stimulation would also suppress other subordinate follicles' growth and induce atresia. Furthermore, studies have also reported that high high-dose exogenous gonadotropins cause chromosomal abnormalities and high aneuploidy rates while stimulating nuclear maturation $[3,13]$.

Although, IVM of immature follicles using less drug stimulation, less cost and more patient-friendly approach compared with conventional $\mathrm{COH}$ in IVF cycle. Recovery of these immature oocytes followed by IVM is still not clear, whether these atresia follicles can be recovered by culturing in vitro or have the ability of folliculogenesis again? Whether the embryos formed by these in vitro mature follicles are healthy or not?

Latest studies in animal models show that the dominant follicle does not adversely affect the developmental and maturational competence of immature bovine oocytes [14], this conclusion has also been verified in human that the development of immature oocytes is not affected either by the presence of a dominant follicle or by the phase of folliculogenesis [15], the results were similar with ours. We performed with 87 embryos from 26 IVF women and 75 embryos from 26 IVM women, the aneuploid rate is higher in IVF embryos, (32.2\% in IVF vs $28.0 \%$ in IVM, respectively) with no significant difference (Table 1). This result was similar to a previous retrospective study in that the frequency of chromosomal abnormality in cleavage stage embryos from IVM and IVF cycles were no significant different [16]. This may be due to the supraphysiological levels of some growth factors and hormones in IVM media, and the less competition for follicles to get these factors in vitro to compare to culture in vivo [17]. A similar result in another study 
suggested that women with premature ovarian insufficiency using IVM of immature follicles can get higher pregnancy and delivery rate and yield better clinical outcomes [18].

We survey the analysis of the aneuploidy frequency in combination with female age showed that aneuploidy rate was increased gradually with women's age. Women below the age of 35 years are nearly $20 \%$, increasing to $32.5 \%$ in 39 years old, and $66.66 \%$ for women more than 40 years. This is similar to the study from Fragouli et al. [19]. Women reaching age 35 years face a decline in fecundability attributed to the steep increase in aneuploidy rates which is reflected by the higher miscarriage rates $[6,20]$.

In this study, the embryos with morphological grade 1 have the lowest aneuploidy frequency (16.6\%) and increase by the grade (Fig. 2d). This was similar to previous studies that observed that the embryos with good quality were more likely to be euploid [21]. Embryos with grade 1 and 2 have a similar rate of aneuploidy both in IVM group and IVF group, but in grade III, embryos in IVM group were more likely to be euploid than IVF group ( $60 \%$ vs $40 \%$, respectively). Due to the pathway in immature follicles were continue to express as well as the expression of genes related to cellular and homeostasis storage, IVM medium has supplementation of the factor for oocyte maturation than in vivo [22].

In conclusion, the results from this study demonstrate that IVM does not affect the quality of embryos, also does not increase the aneuploidy rate of embryos. For women, more than 40 years have a high aneuploidy rate and need to be screened by PGS. An embryo with poor quality also needs to be tested by PGS.

\section{Abbreviations}

ART: Assisted reproductive technology; IVF: In vitro fertilization; IVM: In vitro oocyte maturation; PGS: Preimplantation genetic screening; ICSI: Intracytoplasmic sperm injection; $\mathrm{COH}$ : Controlled ovarian hyperstimulation; OHSS: Ovarian hyperstimulation syndrome; hMG: Human menopausal gonadotrophin.

\section{Acknowledgements}

We thank all the patients who agreed to participate in this study. And we grateful for all the clinical, nursing, embryology, and administrative staff of the reproduction team for their support.

\section{Authors' contributions}

YX, YZL, RCQ conceived and designed the study. JHL, TS, JC, SWZ performed the experiments. SWZ, JHL and TTJ analyzed the data. JHL, JC wrote the manuscript. All authors read and approved the final manuscript.

\section{Funding}

This work was supported by the National Natural Science Foundation of China (81401271).

\section{Availability of data and materials}

The datasets used and analyzed during the current study are available from the corresponding author on reasonable request.

\section{Declarations}

Ethics approval and consent to participate

This study was approved by the Ethics Committee of the Seventh Medical Center of PLA General Hospital.

\section{Consent for publication}

Written informed consent was obtained from each study participant.

\section{Competing interests}

The authors declare that they have no competing interests.

\section{Author details}

${ }^{1}$ Reproductive Medical Center, Senior Department of Obstetrics \& Gynecology, The Seventh Medical Center of PLA General Hospital, Beijing 100700,

China. ${ }^{2}$ Reproductive Medicine Center, The First Affiliated Hospital of Xiamen University, No. 6 Guchengxi Road, Si Ming, Xiamen 361003, China. ${ }^{3}$ Research Group for Reproductive Medicine and IVF Laboratory, Department of Obstetrics and Gynecology, Cologne University, Kerpener Str. 7, 50931 Cologne, Germany. ${ }^{4}$ Reproductive Medical Center, Department of Obstetrics and Gynecology, Peking University International Hospital, Beijing 102206, China. ${ }^{5}$ Center for Reproductive Medicine, Shanghai Tenth People's Hospital of Tongji University, Shanghai 200072, China.

Received: 29 May 2021 Accepted: 14 September 2021

Published online: 09 October 2021

\section{References}

1. Smitz JE, Thompson JG, Gilchrist RB. The promise of in vitro maturation in assisted reproduction and fertility preservation. Semin Reprod Med. 2011;29(1):24-37.

2. Bodri D, Guillén J, Polo A, Trullenque M, Esteve C, Coll O. Complications related to ovarian stimulation and oocyte retrieval in 4052 oocyte donor cycles. Reprod Biomed Online. 2008;17(2):237-43.

3. Baart EB, Martini E, Eijkemans MJ, Van Opstal D, Beckers NG, Verhoeff $A$, et al. Milder ovarian stimulation for in-vitro fertilization reduces aneuploidy in the human preimplantation embryo: a randomized controlled trial. Hum Reprod. 2007;22(4):980-8.

4. Walls ML, Hunter T, Ryan JP, Keelan JA, Nathan E, Hart RJ. In vitro maturation as an alternative to standard in vitro fertilization for patients diagnosed with polycystic ovaries: a comparative analysis of fresh, frozen and cumulative cycle outcomes. Hum Reprod. 2015;30(1):88-96.

5. Lee E, Illingworth P, Wilton L, Chambers GM. The clinical effectiveness of preimplantation genetic diagnosis for aneuploidy in all 24 chromosomes (PGD-A): systematic review. Hum Reprod. 2015;30(2):473-83.

6. Franasiak JM, Forman EJ, Hong KH, Werner MD, Upham KM, Treff NR, et al. The nature of aneuploidy with increasing age of the female partner: a review of 15,169 consecutive trophectoderm biopsies evaluated with comprehensive chromosomal screening. Fertil Steril. 2014;101(3):656-663. e1.

7. Son WY, Tan SL. Laboratory and embryological aspects of hCG-primed in vitro maturation cycles for patients with polycystic ovaries. Hum Reprod Update. 2010;16(6):675-89.

8. XuY, Li J, Zhou G, Guo J. Clinical outcomes for various causes of infertility with natural-cycle in vitro fertilization combined with in vitro maturation of immature oocytes. Fertil Steril. 2010;94(2):777-80.

9. Jiao J, Shi B, Sagnelli M, Yang D, Yao Y, Li W, et al. Minimally invasive preimplantation genetic testing using blastocyst culture medium. Hum Reprod. 2019;34(7):1369-79.

10. Fang R, Yang W, Zhao X, Xiong F, Guo C, Xiao J, et al. Chromosome screening using culture medium of embryos fertilised in vitro: a pilot clinical study. J Transl Med. 2019;17(1):73.

11. Hou Y, Fan W, Yan L, Li R, Lian Y, Huang J, et al. Genome analyses of single human oocytes. Cell. 2013;155(7):1492-506.

12. Bolger AM, Lohse M, Usadel B. Trimmomatic: a flexible trimmer for Illumina sequence data. Bioinformatics. 2014;30(15):2114-20. 
13. Katz-Jaffe MG, Trounson AO, Cram DS. Chromosome 21 mosaic human preimplantation embryos predominantly arise from diploid conceptions. Fertil Steril. 2005;84(3):634-43.

14. Chian RC, Chung JT, Downey BR, Tan SL. Maturational and developmental competence of immature oocytes retrieved from bovine ovaries at different phases of folliculogenesis. Reprod Biomed Online. 2002;4(2):127-32.

15. Chian RC, Buckett WM, Abdul Jalil AK, Son WY, Sylvestre C, Rao D, et al. Natural-cycle in vitro fertilization combined with in vitro maturation of immature oocytes is a potential approach in infertility treatment. Fertil Steril. 2004;82(6):1675-8.

16. Zhang XY, Ata B, Son WY, Buckett WM, Tan SL, Ao A. Chromosome abnormality rates in human embryos obtained from in-vitro maturation and IVF treatment cycles. Reprod Biomed Online. 2010;21(4):552-9.

17. Yu YS, Sui HS, Han ZB, Li W, Luo MJ, Tan JH. Apoptosis in granulosa cells during follicular atresia: relationship with steroids and insulin-like growth factors. Cell Res. 2004;14(4):341-6.

18. Lee HJ, Barad DH, Kushnir VA, Shohat-Tal A, Lazzaroni-Tealdi E, Wu YG, et al. Rescue in vitro maturation (IVM) of immature oocytes in stimulated cycles in women with low functional ovarian reserve (LFOR). Endocrine. 2016;52(1):165-71.
19. Fragouli E, Alfarawati S, Daphnis DD, Goodall NN, Mania A, Griffiths T, et al. Cytogenetic analysis of human blastocysts with the use of FISH, $\mathrm{CGH}$ and aCGH: scientific data and technical evaluation. Hum Reprod. 2011;26(2):480-90.

20. Steiner AZ, Jukic AM. Impact of female age and nulligravidity on fecundity in an older reproductive age cohort. Fertil Steril. 2016;105(6):15841588.e1.

21. Eaton JL, Hacker MR, Harris D, Thornton KL, Penzias AS. Assessment of day-3 morphology and euploidy for individual chromosomes in embryos that develop to the blastocyst stage. Fertil Steril. 2009;91 (6):2432-6.

22. Wells D, Patrizio P. Gene expression profiling of human oocytes at different maturational stages and after in vitro maturation. Am J Obstet Gynecol. 2008;198(4):455.e1-9.

\section{Publisher's Note}

Springer Nature remains neutral with regard to jurisdictional claims in published maps and institutional affiliations.
Ready to submit your research? Choose BMC and benefit from:

- fast, convenient online submission

- thorough peer review by experienced researchers in your field

- rapid publication on acceptance

- support for research data, including large and complex data types

- gold Open Access which fosters wider collaboration and increased citations

- maximum visibility for your research: over 100M website views per year

At BMC, research is always in progress.

Learn more biomedcentral.com/submissions 